\title{
Difference in leg asymmetry between female collegiate athletes and recreational athletes during drop vertical jump
}

Yutaro Morishige ${ }^{1}$, Kengo Harato ${ }^{1,2^{*}}$, Shu Kobayashi ${ }^{1}$, Yasuo Niki ${ }^{1}$, Morio Matsumoto ${ }^{1}$, Masaya Nakamura ${ }^{1}$ and Takeo Nagura, ${ }^{1,2}$

\begin{abstract}
Background: Neuromuscular imbalance will lead to loading asymmetry in sporting activities. This asymmetry is related to leg dominance, which has been associated with increased risk of anterior cruciate ligament (ACL) injury. Therefore, potential biomechanical differences between legs are important. However, little attention has been paid to the biomechanical details of leg dominance. The purpose of the present study was to clarify the relationship between leg dominance and knee biomechanics in females with different activity level during dynamic athletic tasks.
\end{abstract}

Methods: A total of 23 female collegiate (mean age $=19.6 \pm 1.4$ years, mean body mass index $=21.5 \pm 0.9$ ) and 19 recreational athletes (mean age $=20.7 \pm 1.1$ years, mean body mass index $=20.5 \pm 1.7$ ) were enrolled. Tegner activity scores of the collegiate and recreational athletes were 9 and 7, respectively. Knee kinematic and kinetic asymmetries between the dominant (DL) and non-dominant (NDL) legs during the landing phase of drop vertical jump (DVJ) were assessed using three-dimensional motion analysis in collegiate and recreational athletes separately. Statistical comparison was done using two-tailed paired $t$ test between $\mathrm{DL}$ and NDL in each athlete.

Results: The peak knee abduction angle was significantly larger on the DL than on the NDL in collegiate athletes. Knee abduction angle at initial contact (IC), peak knee abduction angle, knee internal rotation angle at IC, and peak knee internal rotation angle were significantly larger on the NDL than on the $\mathrm{DL}$ in recreational athletes. Moreover, peak knee abduction moment within 40 ms from IC was larger on the NDL than on the DL in recreational athletes, while the moment was not significantly different in collegiate athletes.

Conclusions: From the present study, the relationship between leg dominance and knee biomechanics was totally different in females with different activity level. Specifically, asymmetry of the knee abduction angle between limbs was opposite between female recreational and collegiate athletes. According to previous literatures, abduction and internal rotation angles as well as abduction moment were key issues for mechanism of non-contact ACL injury. Therefore, the NDL in female recreational athletes was associated with increased risk of ACL injury.

Keywords: Anterior cruciate ligament, Lower extremity, Non-contact injury, Exercise intensity, Asymmetric motion, Leg dominance, Jump task

\footnotetext{
* Correspondence: kharatoh@yahoo.co.jp

${ }^{1}$ Department of Orthopedic Surgery, Keio University School of Medicine,

Tokyo, Japan

${ }^{2}$ Department of Clinical Biomechanics, Keio University School of Medicine, 35

Shinanomachi, Shinjuku-ku, Tokyo 160-8582, Japan
}

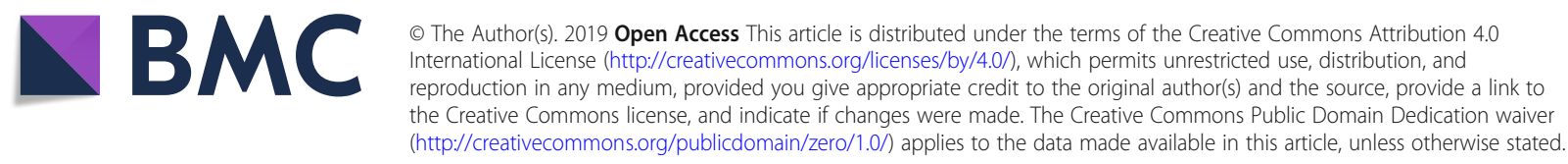




\section{Background}

Leg dominance in sports leads to loading asymmetry and can contribute to the development of unilateral damage to the lower limbs such as anterior cruciate ligament (ACL) injuries, especially in females [1-3]. About 70 to $80 \%$ of ACL tears involve non-contact injuries such as landing from a jump, a quick stop, or a cutting maneuver [4]. Moreover, female athletes were at greater risk of ACL injury than male athletes in non-contact sports [5]. According to previous studies, leg dominance is an important factor in non-contact ACL injuries [6-8]. For instance, Brophy et al. reported that $68 \%$ of females were injured on the non-dominant leg (NDL) and $74 \%$ of males were injured on the dominant leg (DL) in ACL injury during soccer [6]. In addition, Ruedl et al. reported that female recreational skiers suffered more often from non-contact ACL injuries on the NDL than male recreational skiers; however, there was no difference on the DL [8], although conclusions have remained inconsistent. Based on a previous study, physical performance including vertical jump might be reduced by inter-limb asymmetries [9]. For this reason, loading asymmetry has been associated with the intensity and frequency of sporting activities.

Drop vertical jump (DVI) provides a useful screening test for the risk of non-contact ACL injuries in females [10]. The DVJ requires an athlete to drop from a static box, land, and immediately execute a maximal vertical jump. Based on kinematic and kinetic performance traits and anatomical variables, an algorithm has been designed using the DVJ to evaluate the cumulative risk of noncontact ACL injury [10]. The DVJ has thus been used as a method of evaluating the risk of ACL injury in various studies [11-22]. Although, the DVJ is a double-limb landing task, limb asymmetry, which is an established factor for repeated ACL injury, is evident during this test [2]. However, little is known about the effects of asymmetric knee kinematics and kinetics during DVJ in females who participate in sports at different level of intensity.

The purpose of the present study was to clarify the effects of leg dominance on the biomechanics of DVJ among healthy female collegiate and recreational athletes. It was hypothesized that loading condition would be different between female recreational athletes and collegiate athletes during DVJ.

\section{Methods}

\section{Participants}

All participants in the current study were recruited by personal contacts of the authors. The participants in the present study comprised 23 female collegiate athletes (mean age, $19.6 \pm 1.4$ years; height, $1.61 \pm 0.05 \mathrm{~m}$; weight, $56.3 \pm 4.4 \mathrm{~kg}$; basketball players, $n=15$; soccer players, $n=$ 8 ; mean competition history, $7.5 \pm 4.3$ years) and 19 female recreational athletes (mean age, $20.7 \pm 1.1$ years; height,
$1.61 \pm 0.06 \mathrm{~m}$; weight, $53.2 \pm 4.8 \mathrm{~kg}$; basketball players $n=$ 10; volleyball players, $n=9$; mean competition history, 5.5 \pm 3.7 years) (Table 1). Twenty-three female collegiate athletes were part of the Keio University Athletic Association, and 19 female recreational athletes were medical students of Keio University School of Medicine. Tegner activity scores among the collegiate and recreational athletes were 9 and 7, respectively. Based on practice schedule, we observed the actual practice and confirmed that physical demands in collegiate athletes were $3 \mathrm{~h}$ a day, five times a week, and those in recreational athletes were $3 \mathrm{~h}$ a day, three times a week, respectively. The dominant leg was the right side for all participants except for one participant in each group. As female athletes were at greater risk of ACL injury than male athletes in non-contact sports $[1,2]$, females were chosen in the current study. None of the participants had a history of major injury to the trunk or lower extremities. All of the athletes provided written informed consent to participate in this study, which was approved by our institutional review board (\#20080054).

\section{Test procedures}

We defined DVJ as jumping from a $30-\mathrm{cm}$ high box onto force plates positioned $50 \%$ of the height of each athlete away from the box and immediately rebounding on landing into a maximal vertical jump (Fig. 1). The participants were taught how to execute the DVJ and repeated the procedure several times before three trials were recorded. The DL was defined as the leg with which each athlete preferred to kick a ball [6-8]. We placed 46 retro-reflective markers (diameter, $14 \mathrm{~mm}$ ) at standard anatomical landmarks in preparation for DVJ (Fig. 2). The following segments were tracked using three non-collinear infrared markers: two each for the feet, legs, and thighs, and one each for the pelvis and trunk. To define the axes of each of these eight segments, an anatomical model was created by digitizing the following standard bony landmarks: bilateral acromion processes, xiphoid process, suprasternal notch, 7th cervical vertebra, 10th thoracic vertebra, bilateral anterior and posterior superior iliac spines, bilateral iliac crests, bilateral greater trochanters, bilateral lateral and medial epicondyles, bilateral lateral and medial malleoli, bilateral posterior heels, bilateral medial cuneiforms, bilateral great toes, and bilateral heads of the 5th metatarsals. Four additional tracking markers were placed on each of the frontal aspects of the thigh and shank. Calibration markers (bilateral medial epicondyles and medial malleoli) were removed after the standing trial, and only tracking markers were left on the participants throughout all data collection.

\section{Data processing and analysis}

The DVJ was captured using a motion analysis system comprising eight Oqus cameras (Qualisys, Gothenburg, Sweden) at 120 frames/s and two AM6110 force plates 
Table 1 Subject demographics (mean \pm S.D.)

\begin{tabular}{llll}
\hline & Collegiate athletes $(n=23)$ & Recreational athletes $(n=19)$ & $P$ value \\
\hline Age $($ years $)$ & $19.6 \pm 1.4$ & $20.7 \pm 1.1$ & 0.008 \\
Height $(\mathrm{m})$ & $1.61 \pm 0.05$ & $1.61 \pm 0.06$ & 0.650 \\
Weight $(\mathrm{kg})$ & $56.3 \pm 4.4$ & $53.2 \pm 4.8$ & 0.034 \\
Body mass index $\left(\mathrm{kg} / \mathrm{m}^{2}\right)$ & $21.5 \pm 0.9$ & $20.5 \pm 1.7$ & 0.022 \\
Dominant leg (right/left) & $22 / 1$ & $18 / 1$ & 7 \\
Tegner activity level scale & 9 & 7 &
\end{tabular}

aalues obtained using the two-tailed unpaired $t$ test

with a frequency $600 \mathrm{~Hz}$ (Bertec, Columbus, OH, USA). The force plates collected data about ground reaction force (GRF) at $600 \mathrm{~Hz}$ and were synchronized to the camera sampling rate of $120 \mathrm{~Hz}$. The time at initial contact (IC) and at toe-off (TO) from the jump were identified using the GRF. Three-dimensional kinematic, kinetic, and ground reaction force (GRF) data were recorded bilaterally during initial contact (IC) to toe-off (TO). Only data from the third trial were analyzed. The motions of markers were recorded using Track Manager version 2.7 software (Qualysis). We calculated knee kinematics and kinetics using Visual 3D (C-motion Co., Rockville, MD, USA). Knee flexion angle at IC, peak knee flexion angle, knee abduction angle at IC, peak knee abduction angle, knee internal rotation angle at IC, and peak knee internal rotation angle were adopted as kinematic parameters. Vertical ground reaction force (vGRF) (N), peak value of external knee flexion moment (PKFM) within 40 milliseconds (ms) from IC $(\mathrm{Nm} / \mathrm{kg})$, peak value of external knee abduction moment (PKABDM) within $40 \mathrm{~ms}$ from IC $(\mathrm{Nm} / \mathrm{kg})$, and peak value of external knee internal rotation moment (PKIRM) within $40 \mathrm{~ms}$ from IC $(\mathrm{Nm} / \mathrm{kg})$ were adopted

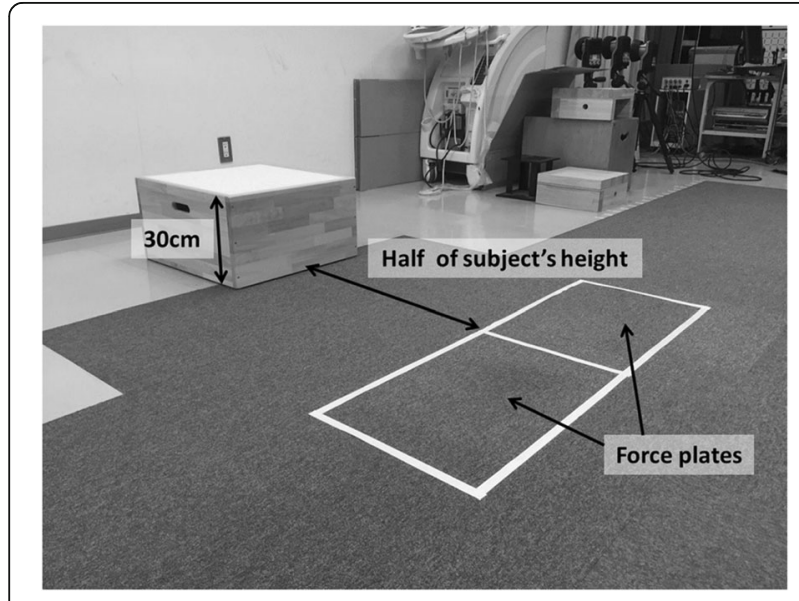

Fig. 1 Setup of the laboratory for drop vertical jump. Drop landing task was defined as jumping from a $30-\mathrm{cm}$ high box to a distance of $50 \%$ of their height away from the box onto force plates and immediately rebounding for a maximal vertical jump on landing

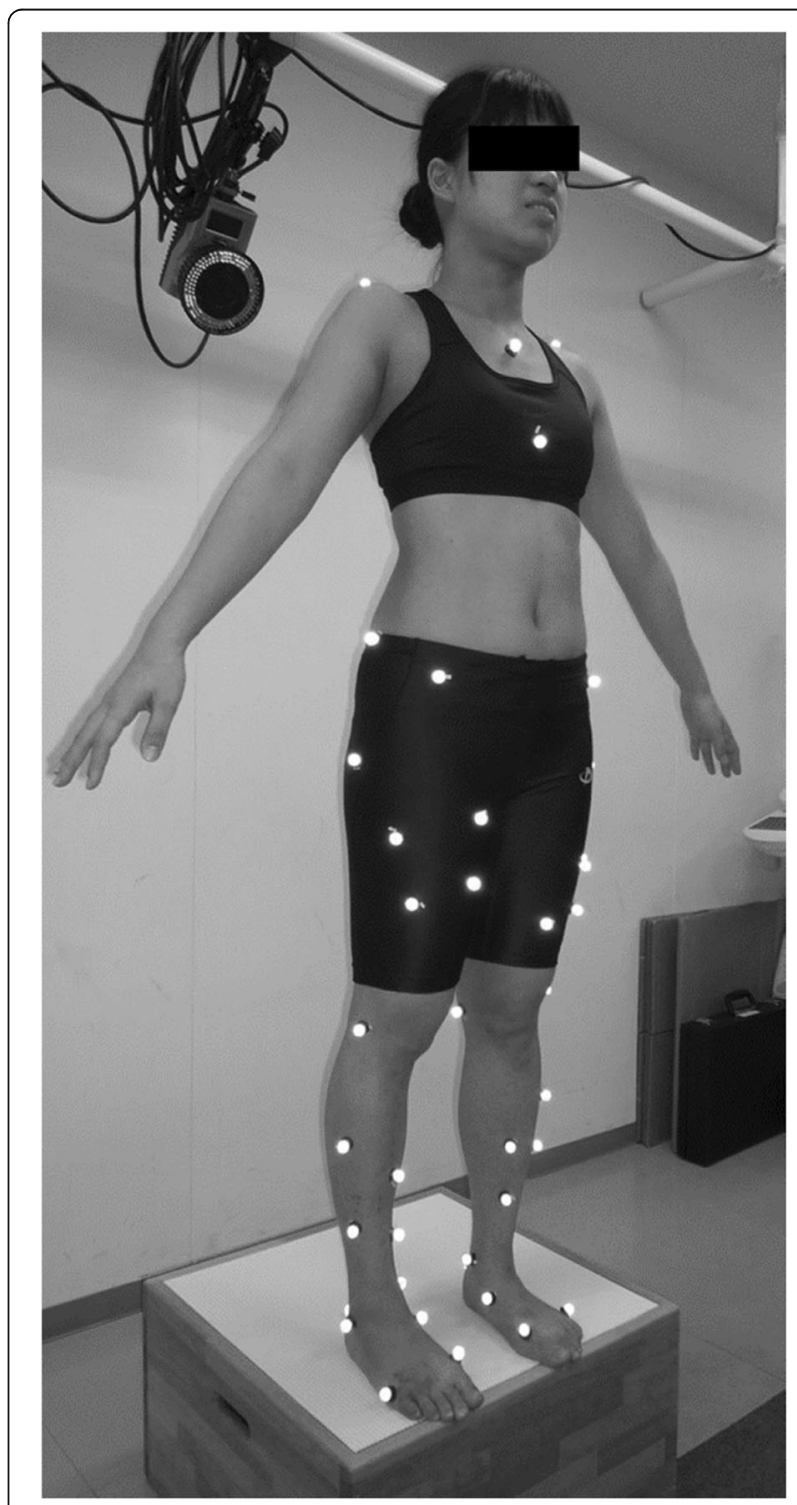

Fig. 2 Maker locations. A total of 46 retro-reflective markers (14 mm in diameter) were placed at standard anatomical landmarks 
as kinetic parameters. Furthermore, an increased risk of non-contact ACL injury was assessed for both limbs in each group. The definition of increased risk was positive value of knee abduction angle at IC and positive value of peak external knee abduction moment within $40 \mathrm{~ms}$ from IC based on previous studies [10-20]. The percentage of limbs with greater risk in each group was evaluated. Biomechanical asymmetry during both bilateral tasks was assessed as the absolute difference between the right and left leg for each data frame.

\section{Statistical analysis}

Demographic data were compared between the female collegiate and recreational athletes using two-tailed unpaired $t$ tests. Kinematic and kinetic data from DVJ were compared between the DL and the NDL within each group using two-tailed paired $t$ tests. All data were statistically analyzed using $\mathrm{SPSS}^{\bullet}$ for Windows software (version 23; Microsoft, Chicago, IL, USA). Values of $P<0.05$ were considered significant. A power analysis was performed using $G^{*}$ Power (v3.1.9.2, Heinrich-Heine University, Düsseldorf, Germany) in each group. Using a large effect size of 0.6 for one-way repeated measures of analysis of variance, a sample size of 19 was required in each group $(\beta=0.80, \alpha=0.05)$.

\section{Results}

The collegiate athletes were significantly younger than the recreational athletes $(P=0.008)$ (Table 1$)$. Similarly, the body mass index (BMI) was significantly larger in female collegiate than recreational athletes $(P=0.034)$.

In terms of kinematics, the knee flexion angle at IC was significantly smaller on the DL than on the NDL $(P=$ 0.005 ) (Table 2), and peak abduction angle was significantly larger on the DL than on the NDL $(P=0.006)$ in collegiate athletes. The knee abduction angle at IC, peak knee abduction angle, knee internal rotation angle at IC, and peak knee internal rotation angle were significantly larger on the NDL than on the DL in female recreational athletes $(P=0.002,<0.001,<0.001$, respectively).

With respect to kinetic parameters, although vGRF did not significantly differ between limbs $(P=0.827)$, PKFM within $40 \mathrm{~ms}$ from IC was significantly smaller on the DL than on the NDL $(P<0.001)$ in collegiate athletes. Similarly, although vGRF did not significantly differ between limbs $(P=0.953)$, KABDM within $40 \mathrm{~ms}$ from IC was notably larger on the NDL than on the DL $(P=$ 0.009 ) in the recreational athletes.

As to the differences between groups, peak knee flexion angle was significantly larger $(P=0.015)$, peak knee internal rotation angle was significantly larger $(P=0.047)$, and PKABM within $40 \mathrm{~ms}$ from IC was significantly larger in collegiate athletes, compared to recreational athletes on the DL. The peak knee flexion angle at IC was significantly larger $(P=0.0039)$, knee abduction angle at IC was significantly smaller $(P=0.0018)$, knee internal rotation angle at IC was significantly smaller $(P<0.001)$, peak knee internal rotation angle was significantly smaller $(P=0.0019)$, and PKFM within $40 \mathrm{~ms}$ from IC was significantly larger $(P=$ $0.024)$ in collegiate athletes, compared to recreational athletes on the NDL.

Concerning the percentage of limbs that were at increased risk in each group, $10.5 \%$ on the DL and $73.7 \%$ on the NDL in female recreational athletes were at increased risk, while $30.4 \%$ on the DL and $21.7 \%$ on the NDL in female recreational athletes were at increased risk for non-contact ACL injury.

\section{Discussion}

The present findings partly supported the hypothesis that loading condition would be different between female

Table 2 Kinematic and kinetic differences between DL and NDL (mean \pm S.D.)

\begin{tabular}{|c|c|c|c|c|c|c|}
\hline & \multicolumn{2}{|c|}{ Collegiate athletes } & \multirow{2}{*}{$\begin{array}{l}P \\
\text { value }^{b}\end{array}$} & \multicolumn{2}{|c|}{ Recreational athletes } & \multirow{2}{*}{$\begin{array}{l}P \\
\text { value }^{c}\end{array}$} \\
\hline & $\overline{\mathrm{DL}}$ & $\mathrm{NDL}$ & & $\overline{\mathrm{DL}}$ & $\mathrm{NDL}$ & \\
\hline Knee flexion angle at IC (deg.) & $33.0 \pm 8.4$ & $35.6 \pm 8.3$ & 0.005 & $35.5 \pm 9.4$ & $35.3 \pm 11.2$ & 0.868 \\
\hline Peak knee flexion angle at IC (deg.) & $107 \pm 12.9$ & $109 \pm 12.6$ & 0.127 & $97.1 \pm 12.6^{d}$ & $96.8 \pm 13.0^{d}$ & 0.791 \\
\hline Knee abduction angle at IC (deg.) & $-2.1 \pm 8.5$ & $-3.9 \pm 6.8$ & 0.143 & $-4.8 \pm 4.8$ & $2.3 \pm 4.9^{e}$ & 0.002 \\
\hline Peak knee abduction angle (deg.) & $5.4 \pm 11.8$ & $1.5 \pm 10.4$ & 0.006 & $1.3 \pm 7.2$ & $6.3 \pm 5.4$ & 0.038 \\
\hline Knee internal rotation angle at IC (deg.) & $-7.6 \pm 10.4$ & $-9.1 \pm 9.1$ & 0.400 & $-12.4 \pm 8.7$ & $10.3 \pm 8.2$ & $<0.001$ \\
\hline Peak knee internal rotation angle (deg.) & $4.4 \pm 6.8$ & $5.5 \pm 6.7$ & 0.557 & $0.0067 \pm 7.0^{d}$ & $11.8 \pm 5.4^{e}$ & $<0.001$ \\
\hline vGRF within 40 ms from IC (N) & $1236 \pm 629$ & $1261 \pm 366$ & 0.827 & $1078 \pm 314$ & $1084 \pm 445$ & 0.953 \\
\hline PKFM within 40 ms from IC (Nm/kg) & $1.69 \pm 0.57$ & $2.71 \pm 0.88$ & $<0.001$ & $1.67 \pm 0.49$ & $2.08 \pm 0.87^{d}$ & 0.068 \\
\hline PKABDM within 40 ms from IC (Nm/kg) & $0.36 \pm 0.31$ & $0.21 \pm 0.36$ & 0.827 & $0.17 \pm 0.24^{d}$ & $0.38 \pm 0.30$ & 0.009 \\
\hline PKIRM within 40 ms from IC ( Nm/kg) & $0.16 \pm 0.16$ & $0.36 \pm 0.39$ & 0.675 & $0.22 \pm 0.15$ & $0.36 \pm 0.39$ & 0.175 \\
\hline
\end{tabular}

${ }^{\mathrm{b}}$ Values obtained using the two-tailed paired $t$ test in collegiate athletes

'Values obtained using the two-tailed paired $t$ test in recreational athletes

${ }^{\mathrm{d} V}$ Values were significantly larger in collegiate athletes than in recreational athletes using the two-tailed unpaired $t$ test

eValues were significantly larger in recreational athletes than in collegiate athletes using the two-tailed unpaired $t$ test 
recreational athletes and collegiate athletes during DVJ. The most important finding in the present study was that the pattern of inter-limb kinematic asymmetry during DVI was opposite between female collegiate and recreational athletes. Specifically, side-to-side asymmetry of the knee abduction angle during DVJ was opposite between female recreational and collegiate athletes.

According to the literature, valgus loading increases ACL force $[23,24]$ and leads to a key factor in the mechanisms of non-contact ACL injury. Hewett et al. reported that an increased knee abduction angle at IC, peak knee abduction angle, and peak knee abduction moment would correlate with increased risk for non-contact ACL injury during DVJ among female athletes [10]. In addition, rapid valgus and internal rotational development within $40 \mathrm{~ms}$ after IC is associated with ACL injury [25-27]. The present findings showed a greater internal rotation angle at IC, a greater peak knee internal rotation angle, and greater knee valgus loading on the NDL in female recreational athletes. As greater valgus loading and internal rotation angle were observed on the NDL, compared to the DL in female recreational athletes, the risk for non-contact ACL can be increased on the NDL in recreational athletes.

Neuromuscular asymmetry is related to primary and secondary non-contact ACL injury [1, 2, 10, 28-31]. For example, female athletes have higher side-to-side asymmetry for hamstring isokinetic torque and the ratio of hamstrings to quadriceps. Moreover, Pappas et al. found greater abduction angle and knee kinematic asymmetry in female than male athletes during forward landings [29]. Clinically, the incidence of non-contact ACL injury based on leg dominance has been reported. According to Brophy et al., $68 \%$ of females were injured on the NDL, while $74 \%$ of males were injured on the DL during soccer [6]. Moreover, Ruedl et al. suggested that female recreational skiers suffered more frequent from noncontact ACL injuries on the NDL than male recreational skiers, yet no difference was detected on the DL [8]. Therefore, the probability of injury on the NDL is higher among female than male recreational athletes. The present findings of female recreational athletes were thus similar to previous clinical studies in terms of increased risk of ACL injury on the NDL. According to a previous study, increased knee abduction angle and moment during DVJ were associated with an increased risk of noncontact ACL injuries [10]. Therefore, the NDL in female recreational athletes had the highest risk of non-contact ACL injury in this study (73.7\%). On the other hand, the female collegiate athletes had greater risk of non-contact ACL injury on the DL, because the flexion moment was asymmetric and the peak knee abduction angle was larger on the DL than on the NDL. Sport-specific strategies to reduce asymmetry should be included in programs to prevent non-contact ACL injury.
Several limitations should be described in the current investigation. First, we could not match age and BMI between the groups. These differences might affect lower limb biomechanics. Second, although the risk of noncontact ACL injury was evaluated, the actual incidence of ACL injury could not be investigated. Third, the athletes participated in various sports, and thus, their background might have affected joint loading in the present study. In fact, Keio University School of Medicine does not have a female soccer team. Lastly, the true reason for biomechanical differences during DVJ between female collegiate and recreational athletes remains unknown, although collegiate athletes are more familiar with the jumping task methods that are commonly practiced among sports compared to recreational athletes. Nonetheless, the present results provide important information regarding the characteristics of female knee kinematics and kinetics with different activity level during jumping tasks.

\section{Conclusion}

Asymmetry during DVJ differed between female collegiate and recreational athletes. Specifically, side-to-side asymmetry of the knee abduction angle and moment during DVJ were greater for female recreational athletes than female collegiate athletes. Therefore, the NDL in female recreational athletes is associated with increased risk of ACL injury. Hence, sport-specific asymmetry reduction should be incorporated into programs as a strategy to prevent injury during participation in recreational and collegiate sports.

\section{Abbreviations \\ ACL: Anterior cruciate ligament; DL: Dominant leg; DVJ: Drop vertical jump; GRF: Ground reaction force; IC: Initial contact; NDL: Non-dominant leg; PKABDM: Peak knee abduction moment; PKFM: Peak knee flexion moment; PKIRM: Peak knee internal rotation moment; TO: Toe-off; VGRF: Vertical ground reaction force}

\section{Acknowledgements}

We acknowledge the contribution of the Keio female basketball team, Keio female soccer team, Keio Medical girls' basketball team, Keio Medical girls' volleyball team, Hiroo Osaki, Tomohiro Yanai, Keita Sonoda, Ryutaro Tanaka, Mayu Minemoto, Masayoshi Kurosawa, Rika Uchiyama, Ryuto Yoshida, Yuki Hoshi, Aiko Sakurai, Satoshi Imai, Ryoji Hayakawa, and Sumi Yamashita for their help.

\section{Authors' contributions \\ YM collected data and prepared the manuscript. KH and TK conceived and designed the study and assisted with the statistical analysis. SK and YN developed the study design and interpretation of data. MN and MM assisted in the coordination of the study and manuscript preparation. All authors} read and approved the final manuscript.

Funding

There is no funding in our work.

Availability of data and materials

The datasets used and/or analyzed during the current study are available from the corresponding author on reasonable request. 


\section{Ethics approval and consent to participate}

This study was approved by the institutional review board of Keio University School of Medicine, and participants provided informed consent to participate.

\section{Consent for publication}

We have consent for publication from every participant in the study.

\section{Competing interests}

The authors declare that they have no competing interests.

Received: 25 December 2018 Accepted: 27 November 2019

Published online: 10 December 2019

\section{References}

1. Hewett TE, Di Stasi SL, Myer GD. Current concepts for injury prevention in athletes after anterior cruciate ligament reconstruction. The American journal of sports medicine. 2013;41(1):216-24.

2. Hewett TE, Ford KR, Hoogenboom BJ, Myer GD. Understanding and preventing ACL injuries: current biomechanical and epidemiologic considerations - update 2010. N Am J Sports Phys Ther. 2010;5(4):234-51.

3. Mokhtarzadeh $\mathrm{H}$, Ewing $\mathrm{K}$, Janssen I, Yeow CH, Brown N, Lee PVS. The effect of leg dominance and landing height on ACL loading among female athletes. J Biomech. 2017;60:181-7.

4. Boden BP, Dean GS, Feagin JA Jr, Garrett WE Jr. Mechanisms of anterior cruciate ligament injury. Orthopedics. 2000:23(6):573-8.

5. Montalvo AM, Schneider DK, Webster KE, Yut L, Galloway MT, Heidt RS Jr, et al. Anterior cruciate ligament injury risk in sport: a systematic review and meta-analysis of injury incidence by sex and sport classification. J Athl Train. 2019:54(5):472-82.

6. Brophy R, Silvers HJ, Gonzales T, Mandelbaum BR. Gender influences: the role of leg dominance in ACL injury among soccer players. Br J Sports Med. 2010;44(10):694-7.

7. Negrete RJ, Schick EA, Cooper JP. Lower-limb dominance as a possible etiologic factor in noncontact anterior cruciate ligament tears. J Strength Cond Res. 2007;21(1):270-3.

8. Ruedl G, Webhofer M, Helle K, Strobl M, Schranz A, Fink C, et al. Leg dominance is a risk factor for noncontact anterior cruciate ligament injuries in female recreational skiers. Am J Sports Med. 2012;40(6):1269-73.

9. Bishop C, Read P, McCubbine J, Turner A. Vertical and horizontal asymmetries are related to slower sprinting and jump performance in elite youth female soccer players. J Strength Cond Res. 2018. https://doi.org/10. 1519/jsc.0000000000002544.

10. Hewett TE, Myer GD, Ford KR, Heidt RS Jr, Colosimo AJ, McLean SG, et al. Biomechanical measures of neuromuscular control and valgus loading of the knee predict anterior cruciate ligament injury risk in female athletes: a prospective study. Am J Sports Med. 2005;33(4):492-501.

11. Chappell JD, Limpisvasti $O$. Effect of a neuromuscular training program on the kinetics and kinematics of jumping tasks. Am J Sports Med. 2008;36(6):1081-6.

12. Earl JE, Monteiro SK, Snyder KR. Differences in lower extremity kinematics between a bilateral drop-vertical jump and a single-leg step-down. J Orthop Sports Phys Ther. 2007;37(5):245-52.

13. Harty CM, DuPont CE, Chmielewski TL, Mizner RL. Intertask comparison of frontal plane knee position and moment in female athletes during three distinct movement tasks. Scand J Med Sci Sports. 2011;21(1):98-105.

14. Joseph M, Tiberio D, Baird JL, Trojian TH, Anderson JM, Kraemer WJ, et al. Knee valgus during drop jumps in National Collegiate Athletic Association Division I female athletes: the effect of a medial post. Am J Sports Med. 2008;36(2):285-9.

15. Kristianslund E, Krosshaug T. Comparison of drop jumps and sport-specific sidestep cutting: implications for anterior cruciate ligament injury risk screening. Am J Sports Med. 2013;41(3):684-8.

16. McLean SG, Fellin RE, Suedekum N, Calabrese G, Passerallo A, Joy S. Impact of fatigue on gender-based high-risk landing strategies. Med Sci Sports Exerc. 2007;39(3):502-14

17. Ford KR, Myer GD, Hewett TE. Valgus knee motion during landing in high school female and male basketball players. Med Sci Sports Exerc. 2003; 35(10):1745-50.

18. Shultz SJ, Nguyen AD, Leonard MD, Schmitz RJ. Thigh strength and activation as predictors of knee biomechanics during a drop jump task. Med Sci Sports Exerc. 2009;41(4):857-66.
19. Malfait B, Dingenen B, Smeets A, Staes F, Pataky T, Robinson MA, et al. Knee and hip joint kinematics predict quadriceps and hamstrings neuromuscular activation patterns in drop jump landings. PloS One. 2016;11(4):e0153737.

20. Beaulieu ML, Palmieri-Smith RM. Real-time feedback on knee abduction moment does not improve frontal-plane knee mechanics during jump landings. Scand J Med Sci Sports. 2014;24(4):692-9.

21. Padua DA, Marshall SW, Boling MC, Thigpen CA, Garrett WE Jr, Beutler Al. The Landing Error Scoring System (LESS) is a valid and reliable clinical assessment tool of jump-landing biomechanics: the JUMP-ACL study. Am J Sports Med. 2009;37(10):1996-2002.

22. Cesar GM, Tomasevicz CL, Burnfield JM. Frontal plane comparison between drop jump and vertical jump: implications for the assessment of ACL risk of injury. Sports Biomech. 2016;15(4):440-9.

23. Lloyd DG, Buchanan TS. Strategies of muscular support of varus and valgus isometric loads at the human knee. J Biomech. 2001:34(10):1257-67.

24. Markolf KL, Burchfield DM, Shapiro MM, Shepard MF, Finerman GA, Slauterbeck JL. Combined knee loading states that generate high anterior cruciate ligament forces. J Orthop Res. 1995;13(6):930-5.

25. Koga H, Nakamae A, Shima $Y$, Iwasa J, Myklebust G, Engebretsen L, et al. Mechanisms for noncontact anterior cruciate ligament injuries: knee joint kinematics in 10 injury situations from female team handball and basketball. Am J Sports Med. 2010;38(11):2218-25.

26. Shin CS, Chaudhari AM, Andriacchi TP. The influence of deceleration forces on $\mathrm{ACL}$ strain during single-leg landing: a simulation study. J Biomech. 2007:40(5):1145-52.

27. Withrow TJ, Huston LJ, Wojtys EM, Ashton-Miller JA. The effect of an impulsive knee valgus moment on in vitro relative $A C L$ strain during a simulated jump landing. Clin Biomech. 2006;21(9):977-83.

28. Palmieri-Smith RM, Lepley LK. Quadriceps strength asymmetry after anterior cruciate ligament reconstruction alters knee joint biomechanics and functional performance at time of return to activity. Am J Sports Med. 2015; 43(7):1662-9.

29. Pappas E, Carpes FP. Lower extremity kinematic asymmetry in male and female athletes performing jump-landing tasks. J Sci Med Sport. 2012;15(1):87-92.

30. Pappas E, Sheikhzadeh A, Hagins M, Nordin M. The effect of gender and fatigue on the biomechanics of bilateral landings from a jump: peak values. J Sports Sci Med. 2007;6(1):77-84.

31. Paterno MV, Schmitt LC, Ford KR, Rauh MJ, Myer GD, Huang B, et al. Biomechanical measures during landing and postural stability predict second anterior cruciate ligament injury after anterior cruciate ligament reconstruction and return to sport. Am J Sports Med. 2010;38(10):1968-78.

\section{Publisher's Note}

Springer Nature remains neutral with regard to jurisdictional claims in published maps and institutional affiliations.

Ready to submit your research? Choose BMC and benefit from:

- fast, convenient online submission

- thorough peer review by experienced researchers in your field

- rapid publication on acceptance

- support for research data, including large and complex data types

- gold Open Access which fosters wider collaboration and increased citations

- maximum visibility for your research: over $100 \mathrm{M}$ website views per year

At $\mathrm{BMC}$, research is always in progress.

Learn more biomedcentral.com/submissions 